\title{
The effect of surface roughness on the adhesion of elastic plates with application to biological systems
}

\author{
B. N. J. Persson a) \\ IFF, FZ-Jülich, 52425 Jülich, Germany \\ S. Gorb \\ MPI für Metallforschung, Heisenbergstrasse 3, D-70569 Stuttgart, Germany
}

(Received 28 July 2003; accepted 4 September 2003)

\begin{abstract}
We study the influence of surface roughness on the adhesion of elastic plates. Most real surfaces have roughness on many different length scales, and this fact is taken into account in our analysis. We consider in detail the case when the surface roughness can be described as a self-affine fractal, and study the plate-substrate pull-off force as a function of the surface roughness. Based on the theoretical results we discuss adhesion in biological systems, focusing on the adhesive pads of lizards. () 2003 American Institute of Physics. [DOI: 10.1063/1.1621854]
\end{abstract}

\section{INTRODUCTION}

In this paper we discuss adhesion of an elastic plate to a hard, randomly rough surface, which has many important applications, e.g., in biological systems. ${ }^{1}$ We calculate the plate-substrate pull-off force under the assumption that there is complete contact in the nominal contact area. We assume that the substrate surface has roughness on many different length scales, and consider in detail the case of self-affine fractal surfaces.

Adhesion of an elastic solid to a rough substrate involves the competition between the (negative) attractive adhesion energy, which results mainly from the regions where the two solids are in atomic contact at the interface, and the (positive) repulsive elastic energy associated with the bending of the surface of the elastic solid so that it comes in direct atomic contact with the substrate. Thus, if $A_{0}$ is the nominal contact area between the solids and $A$ the true atomic contact area, then we define the effective interfacial energy

$$
\gamma_{\mathrm{eff}} A_{0}=\Delta \gamma A-U_{\mathrm{el}} \text {. }
$$

Here, $\Delta \gamma=\gamma_{1}+\gamma_{2}-\gamma_{12}$ is the change in the interfacial energy (per unit area) when perfectly flat surfaces of the two solids are brought into contact, and $U_{\mathrm{el}}$ is the elastic (bending) energy necessary in order to make atomic contact at the interface. In this paper we will assume complete contact between the solids in the nominal contact area so that $A$ $=A_{0}$. The more general problem of partial contact was studied in Ref. 2 for semi-infinite solids. In a future publication we plan to consider the case of partial contact also for the the thin-plate adhesion case.

The theory we develop in this paper can be applied to biological adhesion systems, e.g., to the adhesion pads of flies, beetles, spiders, or lizards. In particular, we focus on the adhesion of the gecko foot pad to surfaces with random roughness. This sems to be a case of "dry" adhesion, where no fluid is injected in the contact area, and is hence a par-

${ }^{\text {a)} E l e c t r o n i c ~ m a i l: ~ b . p e r s s o n @ f z-j u e l i c h . d e ~}$ ticularly simple and well-defined system. ${ }^{3}$ The skin on the gecko foot pad is made of a keratinlike protein with an elastic modulus of order $E \approx 10^{9} \mathrm{~Pa}$. This is much higher than the elastic modulus of rubber, where typically $E \approx 10^{6} \mathrm{~Pa}$. However, even for rubber a relative small surface roughness (of order a few micrometers root-mean-square amplitude) is able to eliminate the adhesion completely, resulting in zero pull-off force. ${ }^{4}$ How, then, is it possible for the lizard to adhere even to a very rough stone wall when the elastic modulus of the pad skin is much higher than that of rubber?

During millions of years of evolution, driven by natural selection, an extremely soft elastic layer has appeared on the lizard pad surface. This layer is built in a hierarchical manner from fibers and plates (see Figs. 1 and 2), which reflects the hierarchical nature of most natural surfaces (to which the lizard must be able to adhere), which have roughness on all length scales, from the macroscopic scale (e.g., the size of the lizard toe pad) down to the atomic scale. Thus, the skin of the lizard pad is covered by a dense layer of fibers or hair (setae) (length $\approx 100 \mu \mathrm{m}$ and width $1 \mu \mathrm{m}$ ). Each of these fibers branches out into about 1000 thinner fibers (length $\sim 10 \mu \mathrm{m}$ and width $\sim 0.1 \mu \mathrm{m}$ ), and each terminal fiber ends with a thin $(5-10 \mathrm{~nm})$ leaflike plate (spatula). This hierarchical construction makes the lizard adhesive system elastically very soft on all relevant length scales (from $\mathrm{mm}$ to $\mathrm{nm})$.

In an earlier paper one of us has studied how the elastic bending energy stored in the setae fiber array systems influence the pull-off force. ${ }^{5}$ The force necessary to remove an individual seta (or spatula) was assumed known (e.g., obtained from experiments). In this paper we focus instead on the binding between the spatula leaflike plate and the substrate.

\section{PULL-OFF FORCE}

Consider an elastic plate (thickness $d$ ) in contact with a rough but nominally flat substrate. The plate is able to bend to follow the substrate roughness wavelength components $\lambda$ that are much larger than the thickness $d$ of the plate. Let us 


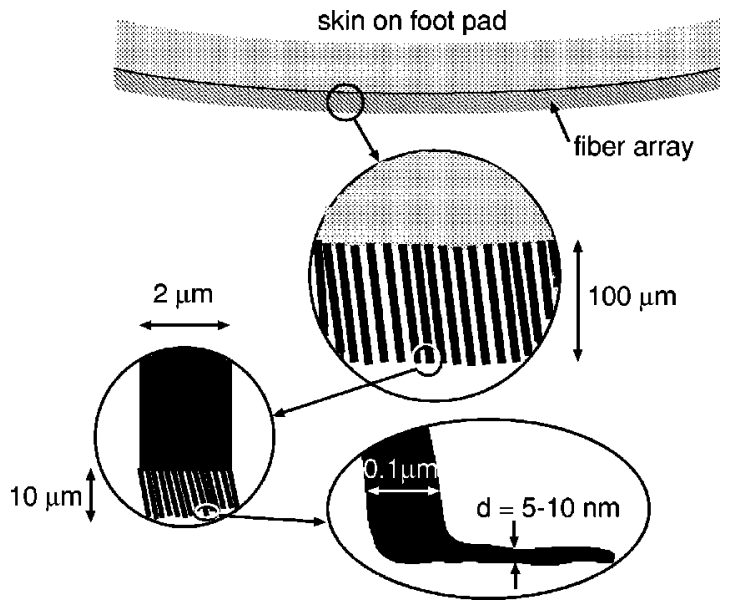

FIG. 1. Schematic picture of the lizard adhesive system. The skin of the lizard is covered by a dense layer of thin fibers or hair (setae) (length $\approx 100 \mu \mathrm{m}$ and width of fiber of order $\sim 1 \mu \mathrm{m}$ ). Each of these fibers branches out into about 1000 thinner fibers (length $\sim 10 \mu \mathrm{m}$ and width of order $\sim 0.1 \mu \mathrm{m})$. Each of the thin fibers ends with a thin $(5-10 \mathrm{~nm})$ leaflike plate (spatula).

first estimate the pull-off force $F$ when the plate is in contact with a smooth (flat) substrate, and the length $l$ of the detached region is large compared to the thickness $d$ of the plate; see Fig. 3(a). The total energy

$$
U=-\Delta \gamma B\left(L_{0}-x\right)+F x(1-\cos \alpha),
$$

where $B$ is the width of the plate and $L_{0}$ the length, and $\Delta \gamma=\gamma_{1}+\gamma_{2}-\gamma_{12}$ the change in surface energy when the plate makes contact with the substrate. The pull-off force is determined by the condition $\partial U / \partial x=0$, which gives

$$
F=\frac{\Delta \gamma B}{1-\cos \alpha} \text {. }
$$

The perpendicular force

$$
F_{\perp}=F \sin \alpha=\frac{\Delta \gamma B \sin \alpha}{1-\cos \alpha} .
$$

Equations (1) and (2) are also valid for rough substrates if we replace the interfacial surface energy difference $\Delta \gamma$ with the effective surface energy $\gamma_{\text {eff }}$ defined in Sec. I. In Fig. 4 we show the (perpendicular) pull-off force as a function of the angle $\alpha$. Note that $F_{\perp} \rightarrow \infty$ as $\alpha \rightarrow 0$. The reader can verify this equation immediately by pulling off a $\operatorname{Scotch}^{\mathrm{TM}}$ tape from a flat substrate at different pulling angles $\alpha$. Equation (1) also gives one reason for why the legs of the lizard point outwards, away from the body; this makes $\alpha$ small and the vertical pull-off force large.

The initial force to "nucleate" the crack at the plate edge [see Fig. 3(b)] is higher than the "steady-state" pull-off force $F$ shown in Fig. 3(a). Thus, if the crack length $l \ll d$ and if the perpendicular stress $\sigma$ act over a region of length $L$ $>d$, we have the standard result

$$
\sigma \approx\left(\frac{E \Delta \gamma}{l}\right)^{1 / 2}
$$

and the pull-off force
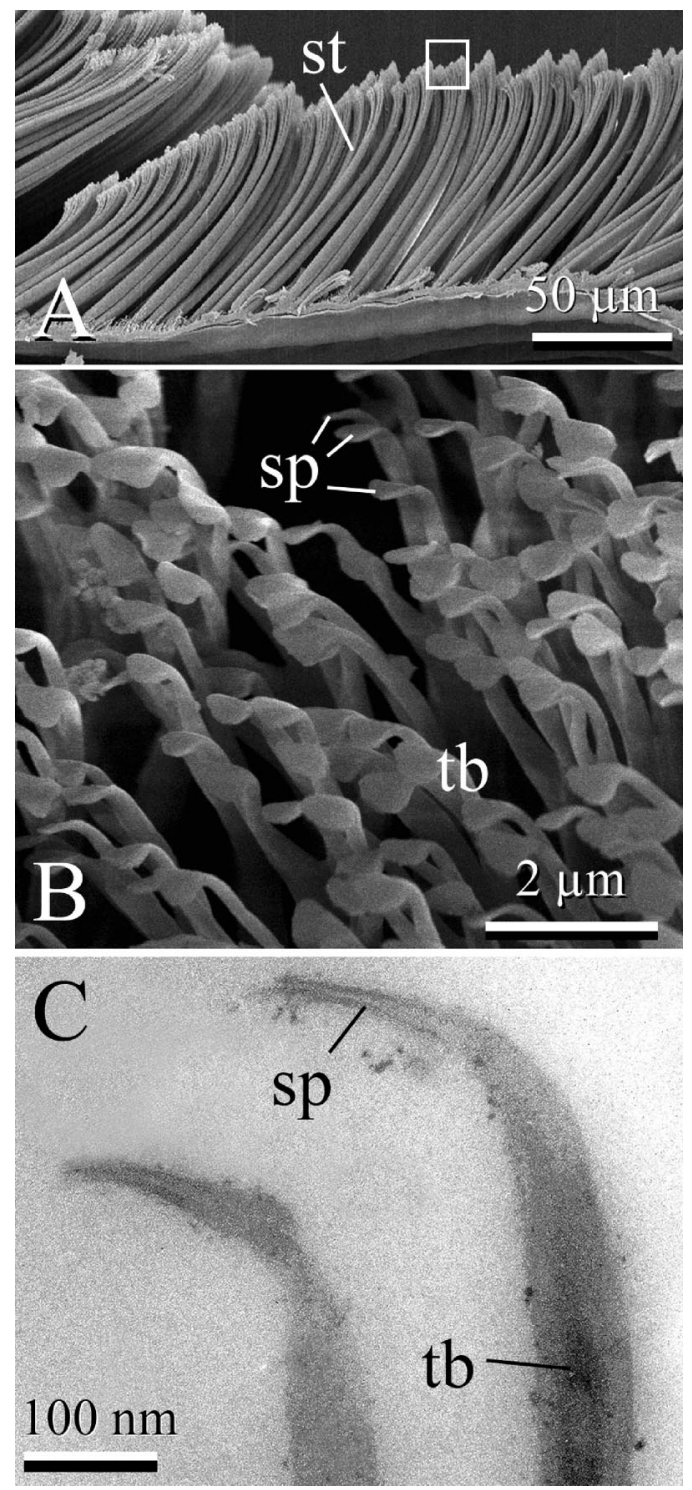

FIG. 2. Details of attachment system of the tokay gecko (Gekko gecko). (A) Scanning electron microscopy (SEM) micrograph of setae (st) located on thin keratin film. (B) Magnification (SEM micrograph) of the area surrounded by the white rectangle in $(\mathrm{A})$, showing terminal branches $(\mathrm{tb})$ of setae with the spatula (sp). (C) Transmission electron microscopy micrograph of ultrathin section of two terminal branches (tb) with spatulae (sp).

$$
F=B L \sigma \approx B L\left(\frac{E \Delta \gamma}{l}\right)^{1 / 2}
$$

The spatula ends with an elastic leaflike plate with the lateral dimensions of order $200-300 \mathrm{~nm}$, and with a thickness which varies from $d \approx 20 \mathrm{~nm}$ at the base to $d \approx 5 \mathrm{~nm}$ at the tip [see Fig. 3(c)]. For a smooth substrate the spatula is likely to adhere along its full length as indicated in the figure. In this case the force necessary in order to initiate pulloff is given by a formula similar to Eq. (2), with $B \approx L \approx D$ of order the thickness $D \approx 0.1 \mu \mathrm{m}$ of the terminal branch, and with an initial crack length equal to some small fraction of the diameter of the terminal branch, e.g., $l \approx 0.1 D$. Using $\Delta \gamma \approx 2 \mathrm{meV} / \AA^{2}$ and $E \approx 10^{9} \mathrm{~Pa}$, this gives the pull-off force of order $1 \mu \mathrm{N}$, which is close to the observed value for smooth substrates. On a rough substrate the interfacial free- 

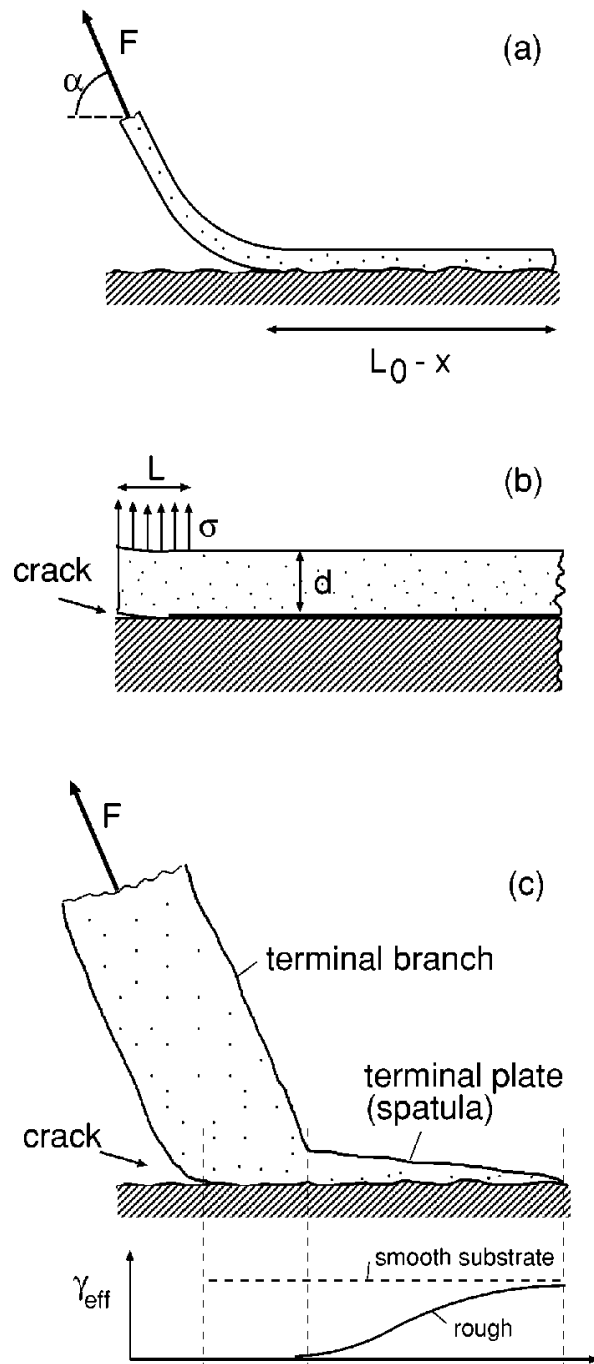

FIG. 3. (a) A thin plate pulled off a substrate (pull-off force $F$ ). (b) The initial force, when the length $l$ of the crack is much shorter than the thickness $d$ of the plate, is much higher than the force in (a) where $l \gg d$. (c) A thin fiber in contact with a relatively smooth substrate. The fiber ends with a thin, platelike structure (spatula) (thickness of order 5-20 nm) which is able to deform to follow the substrate roughness profile. The effective interfacial surface energy, $\gamma_{\text {eff }}$, depends on the thickness of the terminal plate, and we expect $\gamma_{\text {eff }}$ to vary with the location along the spatulae as indicated by the solid line in the bottom part of the figure.

energy difference $\Delta \gamma$ must be replaced by the effective free energy $\gamma_{\text {eff }}$, which includes the elastic energy stored at the interface. The latter depends on the thickness of the terminal plate, and we expect $\gamma_{\text {eff }}$ to vary with the location along the spatula as indicated in Fig. 3(c) (bottom, solid line). Thus, in this case effective adhesion may only occur close to the tip of the terminal plate, and the pull-off force will be determined by this contact region by a formula of the type given by Eq. (1), but with $\Delta \gamma$ replaced by $\gamma_{\text {eff }}$.

\section{INTERFACIAL ELASTIC AND ADHESION ENERGIES FOR ROUGH SURFACES}

Assume that a thin elastic slab (thickness $d$ ) is in contact with the rough surface of a hard solid and that, because of

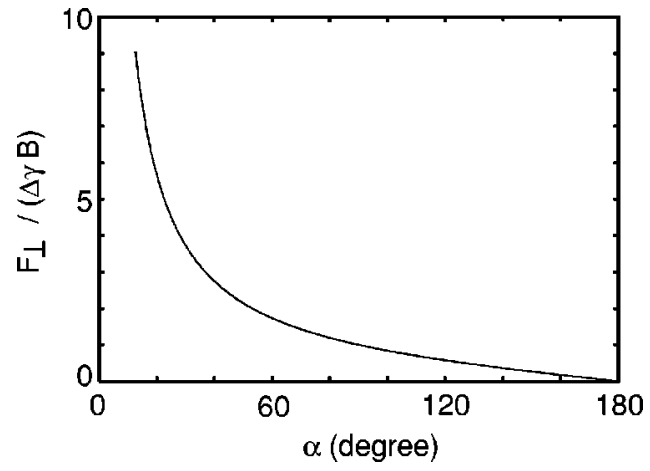

FIG. 4. The perpendicular pull-off force as a function of the angle $\alpha$ defined in Fig. 3.

the slab-substrate adhesion interaction, the slab deforms elastically and makes contact with the substrate everywhere; see Fig. 5.

Let us calculate the difference in the free energy between the slab in contact with the substrate and the noncontact case. Let $z=h(\mathbf{x})$ denote the height of the rough surface above a flat reference plane (chosen so that $\langle h\rangle=0$ ). We assume first that the elastic slab is in direct contact with the substrate over the whole nominal contact area. Let us calculate the elastic energy stored in the deformation field in the elastic slab. We first assume that the thickness $d$ of the slab is much smaller than the shortest wavelength $\lambda$ associated with the substrate roughness profile. In this case we can use the theory of elastic plates to calculate the elastic energy. Let $z=u(\mathbf{x})$ denote the vertical displacement field of a thin plate, which originally (in the undeformed state) occupies the $x-y$ plane. The elastic energy in the plate is given by: ${ }^{6}$

$$
U_{\mathrm{el}}=\frac{E d^{3}}{24\left(1-\nu^{2}\right)} \int d^{2} x\left[\left(\nabla^{2} u\right)^{2}-2(1-\nu)\left|u_{i j}\right|\right],
$$

where the determinant

$$
\left|u_{i j}\right|=\frac{\partial^{2} u}{\partial x^{2}} \frac{\partial^{2} u}{\partial y^{2}}-\left(\frac{\partial^{2} u}{\partial x \partial y}\right)^{2} .
$$

Writing

$$
u(\mathbf{x})=\int d^{2} q u(\mathbf{q}) e^{i \mathbf{q} \cdot \mathbf{x}},
$$

we get

$$
\int d^{2} x\left(\nabla^{2} u\right)^{2}=(2 \pi)^{2} \int d^{2} q q^{4}|u(\mathbf{q})|^{2},
$$

and

$$
\int d^{2} x\left|u_{i j}\right|=0 .
$$

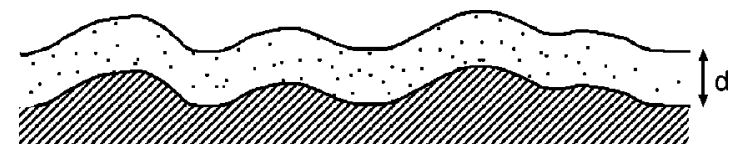

FIG. 5. The adhesion interaction pulls the elastic slab into complete contact with the rough substrate surface. 
(a)

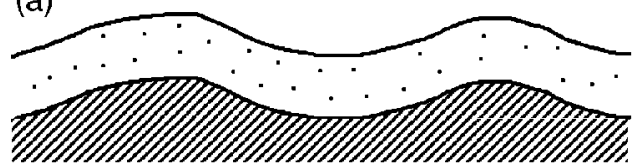

(b)

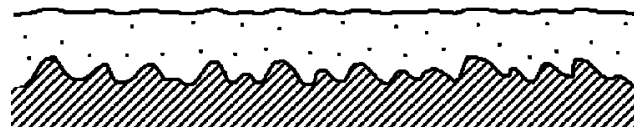

FIG. 6. (a) When the wavelength $\lambda_{0}$ of the surface roughness is much longer than the thickness $d$ of the elastic slab, $\lambda_{0} \gg d$, the elastic slab deforms so that the upper surface of the slab takes the same form as the substrate roughness profile. (b) When $\lambda_{0}<d$ the upper surface of the slab is nearly flat (the displacement field decays as $u \sim \exp \left(-2 \pi z / \lambda_{0}\right)$ with the distance $z$ away from the substrate surface).

For complete contact $u(\mathbf{x})=h(\mathbf{x})$ and hence $u(\mathbf{q})=h(\mathbf{q})$. Now, let us define the surface roughness power spectrum

$$
C(q)=\frac{1}{(2 \pi)^{2}} \int d^{2} x\langle h(\mathbf{x}) h(\mathbf{0})\rangle e^{-i \mathbf{q} \cdot \mathbf{x}},
$$

where $\langle\ldots\rangle$ stands for ensemble average. Note that

$$
\left\langle|h(\mathbf{q})|^{2}\right\rangle=\frac{A_{0}}{(2 \pi)^{2}} C(q),
$$

where $A_{0}$ is the (one side) surface area of the slab. Using (7) and that $u(\mathbf{q})=h(\mathbf{q})$, and substituting (4) and (5) in (3) gives

$$
U_{\mathrm{el}}=\frac{A_{0} E}{24\left(1-\nu^{2}\right)} \int d^{2} q(q d)^{3} q C(q) .
$$

If we now assume that $\lambda \ll d$, we can treat the elastic slab as infinitely thick when deriving the elastic energy stored in the slab (see Fig. 6). If we again assume that complete contact occurs between the solids, then $u_{z}=h(\mathbf{x})$, and as shown in Refs. 2 and 7,

$$
U_{\mathrm{el}}=\frac{A_{0} E}{4\left(1-\nu^{2}\right)} \int d^{2} q q C(q) .
$$

We can interpolate smoothly between the results (8) and (9) using the following expression for the elastic energy:

$$
U_{\mathrm{el}}=\frac{A_{0} E}{4\left(1-\nu^{2}\right)} \int d^{2} q q C(q) \frac{(q d)^{3}}{6+(q d)^{3}} .
$$

The adhesion energy is assumed to be proportional to the contact area, so that (assuming complete contact)

$$
U_{\mathrm{ad}}=-\Delta \gamma A_{0} \text {. }
$$

The change in the free energy when the elastic slab moves in contact with the substrate is given by the sum of (10) and (11)

$$
U_{\mathrm{el}}+U_{\mathrm{ad}}=-\gamma_{\mathrm{eff}} A_{0},
$$

where

$$
\gamma_{\mathrm{eff}}=\Delta \gamma\left[1-\frac{2 \pi}{\delta} \int d q q^{2} C(q) \frac{(q d)^{3}}{6+(q d)^{3}}\right],
$$

where we have introduced the adhesion length $\delta=4(1$ $\left.-\nu^{2}\right) \Delta \gamma / E$. The theory above is valid for surfaces with arbitrary random roughness, but it will now be applied to (a) surfaces with roughness on a single length scale; (b) selfaffine fractal surfaces; and (c) a sandpaper surface for which the power spectra $C(q)$ has been calculated from the measured height profile $h(\mathbf{x})$.

(a) One length scale: Assume surface roughness on a single length scale $\lambda_{0}$. This limiting case is not very realistic, but is very useful in order to understand some aspect of adhesion. We take

$$
C(q)=C_{0} \delta\left(q-q_{0}\right),
$$

where $q_{0}=2 \pi / \lambda_{0}$. We can relate $C_{0}$ to the root-meansquare (rms) roughness amplitude using (6)

$$
\left\langle h^{2}\right\rangle=\int d^{2} q C(q)=2 \pi C_{0} q_{0} .
$$

Following earlier studies we define $h_{0}^{2}=2\left\langle h^{2}\right\rangle$ so that

$$
C_{0}=h_{0}^{2} /\left(4 \pi q_{0}\right) \text {. }
$$

Substituting (14) in (13) and using (15) gives

$$
\gamma_{\mathrm{eff}}=\Delta \gamma\left[1-\left(q_{0} h_{0}\right)^{2} \frac{1}{2 q_{0} \delta} \frac{\left(q_{0} d\right)^{3}}{6+\left(q_{0} d\right)^{3}}\right] .
$$

(b) Self-affine fractal surface: It has been found that many "natural" surfaces, e.g., the surfaces of many materials generated by fracture, can be approximately described as selfaffine surfaces over a rather wide roughness size region. A self-affine fractal surface has the property that if we make a scale change that is appropriately different along the two directions, parallel and perpendicular, then the surface does not change its morphology. ${ }^{8}$ Recent studies have shown that even asphalt road tracks (of interest for rubber friction) are (approximately) self-affine fractal, with a long-distance cutoff length $\lambda_{0}=2 \pi / q_{0}$ of order a few mm. For a self-affine fractal surface ${ }^{8}$ for $q>q_{0}$

$$
C(q)=\frac{H}{2 \pi}\left(\frac{h_{0}}{q_{0}}\right)^{2}\left(\frac{q}{q_{0}}\right)^{-2(H+1)},
$$

where $H=3-D_{\mathrm{f}}$ (where the fractal dimension $2<D_{\mathrm{f}}<3$ ), and where $q_{0}$ is the lower cutoff wave vector. For $q<q_{0}$ we take for simplicity $C(q)=0$. The parameter $h_{0}$ determines the rms roughness amplitude, $\left\langle h^{2}\right\rangle=h_{0}^{2} / 2$. We note that $C(q)$ can be measured directly, using many different methods, including stylus instruments and optical instruments.

Substituting (17) in (13) gives

$$
\frac{\gamma_{\mathrm{eff}}}{\Delta \gamma}=1-\left(q_{0} h_{0}\right)^{2} \frac{1}{q_{0} \delta} f(H),
$$

where

$$
f(H)=H \int_{1}^{q_{1} / q_{0}} d x \frac{\left(q_{0} d\right)^{3} x^{3-2 H}}{6+\left(q_{0} d\right)^{3} x^{3}} .
$$

The short distance cut wave vector cutoff $q_{1}$ depends on the system under study. If it is assumed that the substrate is self-affine fractal on all length scales, then $q_{1} \approx 2 \pi / a$, where $a$ is of order a substrate lattice spacing, i.e., of order a few 


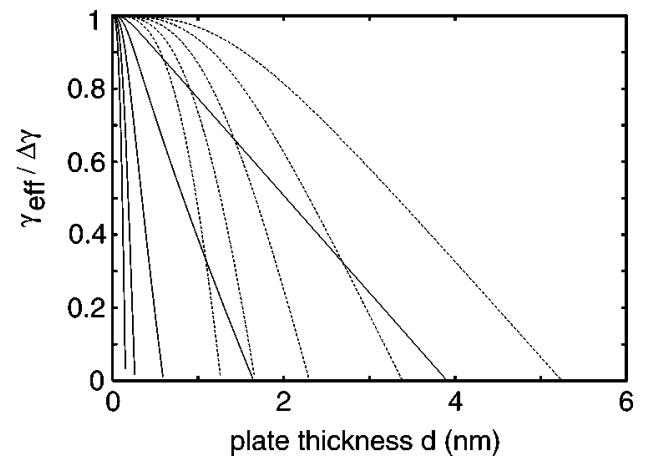

FIG. 7. The variation of the (normalized) effective interfacial free energy $\gamma_{\text {eff }}$ with the thickness $d$ of the plate. Results are shown for $q_{1}=10^{9}$ (dashed lines) and $q_{1}=10^{10} \mathrm{~m}^{-1}$ (solid lines) for $H=0.5,0.6, \ldots, 1$, where the magnitude of $\gamma_{\text {eff }}$ monotonically increases with increasing $H$. In the calculation $\delta=1 \AA$.

angstrom. Thus, the largest possible $q_{1}$ is $\approx 10^{10} \mathrm{~m}^{-1}$. However, if the elastic solid has a thin, very soft (say, liquidlike) layer at its surface, as one of us has speculated before may be the case for the the lizard foot pad, then the effective cutoff wave vector $q_{1}$ will be smaller. For example, if a $D \sim 60 \AA$ high mobility (liquidlike) layer occurs then one expects $q_{1}$ $\approx 2 \pi / D \approx 10^{9} \mathrm{~m}^{-1}$. Similarly, if a thin (thickness $D$ ) (typically organic) contamination layer occurs on the surface, which is able to rearrange itself at the interface and fill out nanoscale cavities, then again $q_{1} \approx 2 \pi / D$. In some cases (e.g., for flies, beetles, and other insects) a liquid substance injected into the contact area will have a similar effect of acting as a large wave vector cutoff in the $q$-integration in Eq. (19).

Let us apply Eq. (18) to the adhesion of a lizard toe to a rough substrate. The elastic modulus of keratin is in the range 1-4 $\mathrm{GPa}$ and, assuming the typical van der Waals surface energy difference, ${ }^{9} \quad \Delta \gamma \approx 1-3 \mathrm{meV} / \AA^{2}$ gives $\delta$ $\approx 1 \AA$. In Fig. 7 we show the calculated [from (18) and (19)] effective surface energy for a typical case. We have used $h_{0}=20 \mathrm{~nm}$, which was obtained from the measured height profile of a sandpaper (the measurement refers to a linear dimension $\lambda_{0}$ of order a few hundred nanometers, i.e., to the lateral size of the leaflike structure at the end of the spatula). We also used $q_{0}=2 \pi / \lambda_{0} \approx 10^{7} \mathrm{~m}^{-1}$ and $q_{1}=10^{9}$ (dashed lines) and $10^{10} \mathrm{~m}^{-1}$ (solid lines). Since the spatula end consists of a platelike structure with an average thickness of $d$ $\approx 5-10 \mathrm{~nm}$, it is clear that in the typical case of $H=0.8$ the adhesion will be suppressed strongly (see also below). However, there is considerable uncertainty in the value of $\delta$ (we have used $\delta=1 \AA$ ), since the elastic modulus $E$ and the interfacial energy difference $\Delta \gamma$ have not been measured accurately until now. Similarly, the thickness of the leaf plate will vary from a maximum at the basis, to a smaller value close to the periphery of the plate.

(c) Sandpaper: Finally, let us present some numerical results for a sandpaper surface for which we plan to study gecko adhesion in the near future. We have measured the height distribution $h(\mathbf{x})$ (over a rectangular area $L_{x} \times L_{y}$ ) using an atomic force microscope (AFM). Using a recently developed computer program ${ }^{10}$ we have obtained the surface

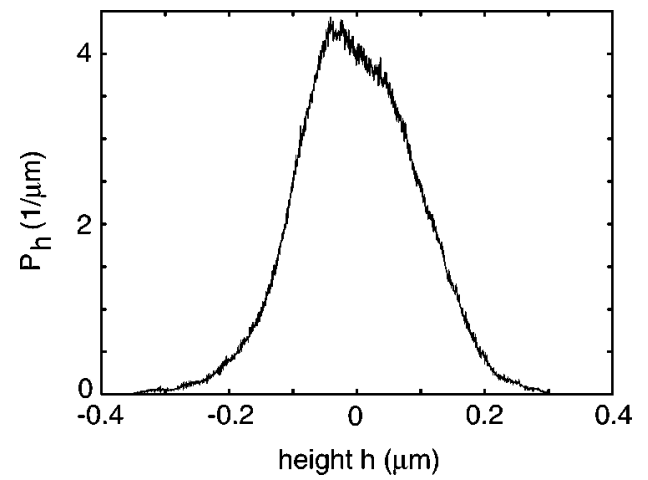

FIG. 8. The height probability distribution $P_{h}$ for a sandpaper (particle diameter $\approx 0.3 \mu \mathrm{m}$ ) surface with a root-mean-square roughness of about 93 $\mathrm{nm}$.

height distribution $P_{h}$ and the surface roughness power spectra $C(q)$ from the height data.

In Fig. 8 we show the height probability distribution $P_{h}$ for the sandpaper surface with particle size of order $0.3 \mu \mathrm{m}$. The root-mean-square roughness $93 \mathrm{~nm}$ was measured over a surface area of the linear size $\lambda_{0} \approx 30 \mu \mathrm{m}$. Note that $P_{h}$ is a near-perfect Gaussian, and one can show that randomly rough surfaces have Gaussian height distributions. Figure 9 shows the surface roughness power spectra $C(q)$ for the same surface. The height profile was measured with a lateral resolution $a=29.3 \mathrm{~nm}$, corresponding to the wave vector $q$ $\approx \pi / a \approx 10^{8} \mathrm{~m}^{-1}$; in Fig. 9 we have made a linear extrapolation to larger $q$ vectors. Note that $C(q)$ has a power law region (i.e., a linear region between $\log C$ and $\log q$ ), characterized by the exponent $H \approx 1.1$.

Figure 10 shows the variation of the (normalized) effective interfacial free energy $\gamma_{\text {eff }}$ with the thickness $d$ of the plate. Curves (a)-(d) correspond to different (short distance) cutoff wave vectors $q_{1}$, namely (a) $10^{7}$; (b) $10^{8}$; (c) $10^{9}$; and (d) $10^{10} \mathrm{~m}^{-1}$. In the calculation we have used the power spectra $C(q)$ shown in Fig. 9, and $\delta=1 \AA$. It is interesting to note that using the large wave vector cutoff $q_{1}=10^{9}$ or $10^{10} \mathrm{~m}^{-1}$ gives nearly the same result. This implies, e.g., that at least in the present case a very soft thin (nanometers) layer at the interface will not increase the adhesion to any appreciable extent, i.e., such a layer may not be necessary in order for the lizard to adhere to rough substrates.

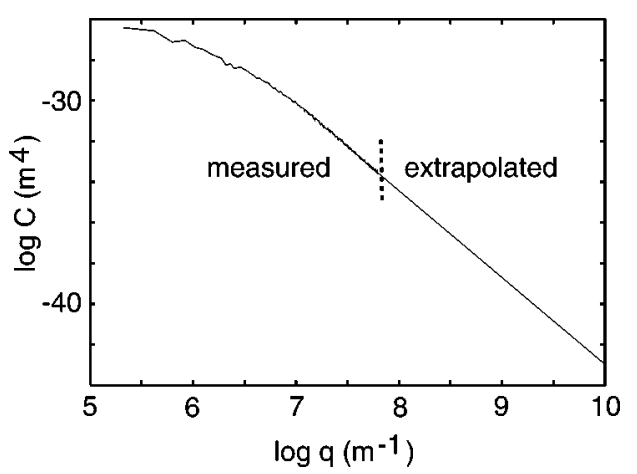

FIG. 9. Surface roughness power spectra $C(q)$ as a function of the wave vector $q$ for the same sandpaper surface as in Fig. 8 . 


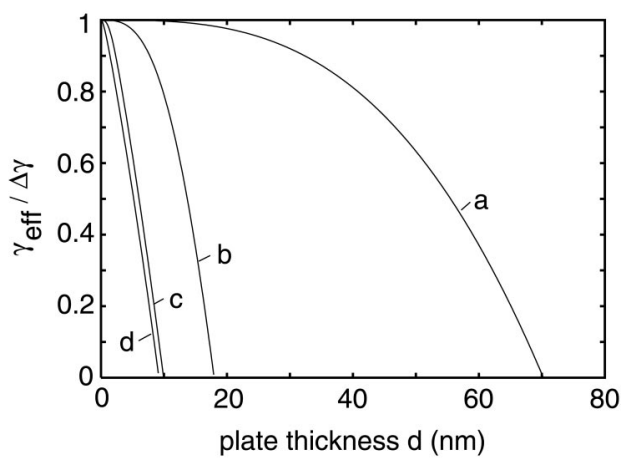

FIG. 10. The variation of the (normalized) effective interfacial free energy $\gamma_{\text {eff }}$ with the thickness $d$ of the plate. Curves (a)-(d) correspond to different short distance cutoff wave vector $q_{1}$, namely (a) $10^{7}$; (b) $10^{8}$; (c) $10^{9}$; and (d) $10^{10} \mathrm{~m}^{-1}$, for the power spectra $C(q)$ shown in Fig. 9 and with $\delta=1 \AA$.

The curves in Fig. 10 were calculated using Eq. (13) with the power spectra $C(q)$ shown in Fig. 9, which start at $q_{0} \approx 2.1 \times 10^{5} \mathrm{~m}^{-1}$. However, when studying the adhesion between the lizard leaflike plate and the substrate we should only include roughness components with wavelengths shorter than the lateral size of the leaf plate, which is of order $\lambda_{0} \approx 300 \mathrm{~nm}$. In Fig. 11 we compare the results from Fig. 10 [curves (b)-(d)] with the effective surface energy obtained when we only include roughness wave vector components with $q>q_{0}=2 \pi / \lambda_{0} \approx 2 \times 10^{7} \mathrm{~m}^{-1}$ (dashed lines). The thickness of the platelike structure at the end of the spatula is in the range of $d \approx 5-10 \mathrm{~nm}$. Based on Fig. 11 [curve (d)], one would expect to observe a strong decrease in the adhesion on this surface as compared to a perfectly smooth substrate (see also Sec. IV). Experiments to check these predictions are in progress. A detailed comparison between theory and experiment would, however, require that the height profile $h(\mathbf{x})$ be measured with higher resolution $(\sim 3 \mathrm{~nm})$ so that $C(\mathbf{q})$ can be calculated at least up to $q \sim 10^{9} \mathrm{~m}^{-1}$, rather than extrapolated to large $q$ as in the present case (see Fig. 9).

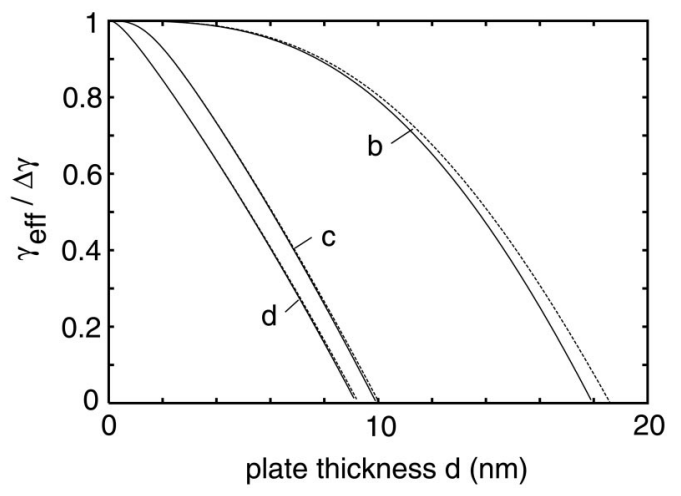

FIG. 11. The variation of the (normalized) effective interfacial free energy $\gamma_{\text {eff }}$ with the thickness $d$ of the plate. Curves (b)-(d) correspond to different short distance cutoff wave vectors $q_{1}$, namely (b) $10^{8}$; (c) $10^{9}$; and (d) $10^{10} \mathrm{~m}^{-1}$, for the power spectra $C(q)$ shown in Fig. 9 and with $\delta=1 \AA$. The solid curves are the same as in Fig. 10, while the dashed curves are obtained by only including roughness wavelength components $q>q_{0}=2$ $\times 10^{7} \mathrm{~m}^{-1}$

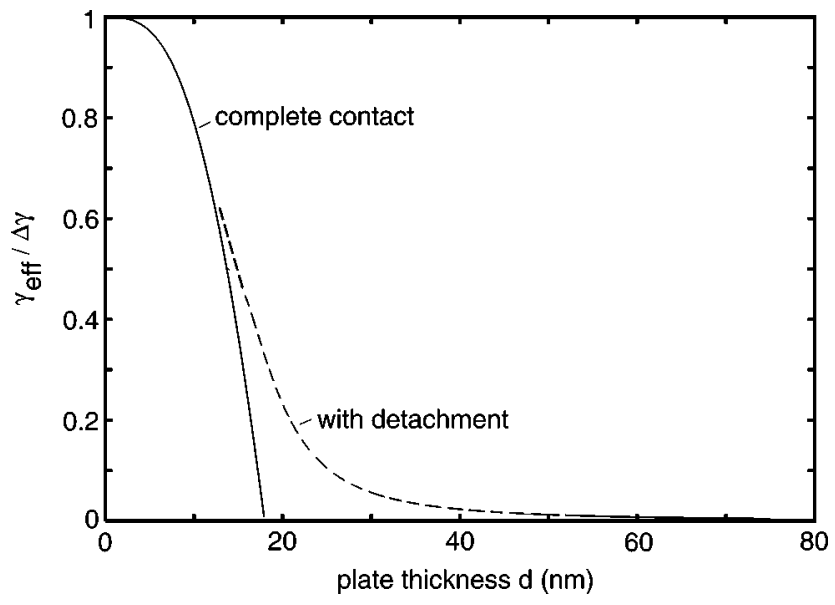

FIG. 12. When detached regions are included, the effective interfacial energy as a function of the thickness $d$ of the slab will have a tail toward larger $d$. For the case (b) in Fig. 10 (schematic).

\section{COMMENTS}

In this section we make two comments related to the theory above. First, we note that when detached regions are included, the effective interfacial energy as function of the thickness $d$ of the slab will have a tail toward larger $d$. This is shown schematically in Fig. 12 (dashed line) for case (b) in Fig. 10. We shall study this effect in detail in the future by generalizing the theory of Ref. 2, which is valid for semiinfinite solids rather than plates.

However, even when the minimum free-energy state corresponds to complete contact, the elastic plate may (because of friction) be trapped in a metastable state as illustrated in Fig. 13. In this case, because the kinetic friction is smaller than the static friction, sliding or vibrating the plate may increase the contact area. This effect is known experimentally: By sliding the lizard toe pad for a short distance the adhesion force can be increased. ${ }^{3}$

Measurements have shown that the friction between the adhesive pad of a beetle (Gastrophysa viridula), and the sandpaper surface discussed in Sec. III(c) is about 5 times smaller than on the smooth substrate (of the same material). One interpretation of this result is that the area of real contact may be $\sim 5$ times smaller on the rough substrate compared with the smooth substrate. The theory presented in this paper assumes complete contact, but when the theory predicts $\gamma_{\text {eff }} / \Delta \gamma \ll 1$ (as in Sec. III) one may, in fact, expect only partial contact (see above); this would be consistent with the beetle friction data. When sandpaper with larger particles

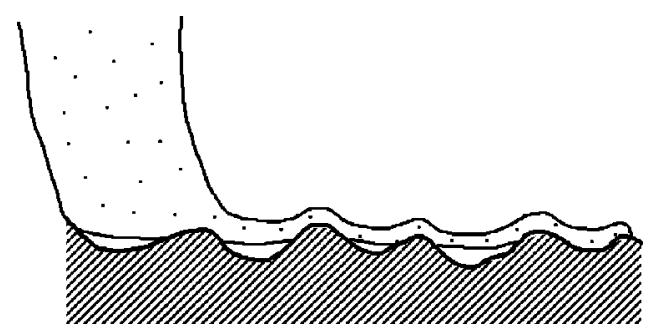

FIG. 13. The free energy is minimal for the complete contact state, but because of friction the plate is not able to deform to follow the substrate. 
was used $(>1 \mu \mathrm{m})$ the friction was nearly the same as on the smooth substrate, indicating complete contact in these cases. Unfortunately, no AFM data of the height profile were reported for these cases.

It has been pointed out that on a smooth surface only $0.03 \%$ of the gecko's setae are necessary in order to support its body weight, and the question has been raised why the geckos are so overbuilt. ${ }^{3}$ However, it is clear from the calculations presented in Sec. III and from Fig. 12 that on a rough substrate the spatulae-substrate adhesion may be strongly reduced, and we believe that this may be the main reason why the gecko's adhesive system is so apparently overbuilt.

\section{DISCUSSION}

How can a fly or a cricket walk on a glass window, or a lizard move on a stone or concrete wall? In order to explain the observed adhesion, these questions can be reformulated as follows: how is the extremely soft surface layer, which must exist on the adhesion pads, designed? This fundamental question has interested scientists for many years, and recently very important work has been performed in order to gain a deeper insight into this problem. ${ }^{1}$ Thus, it is now known that the adhesive systems, adapted to attachment to a variety of surfaces, are built in a hierarchical manner from fibers and plates with very small bending elasticities, making it possible for the molecular attraction at the interface to pull the two surfaces into nearly complete contact without storing a large elastic deformation energy at the interface.

In this paper we have focused on dry adhesion, which seems to be relevant for lizards. ${ }^{3}$ In Ref. 5 one of us presented a simple model study of fiber adhesion on surfaces with roughness on many length scales, and applied it to the adhesion between a lizard toe and a smooth or rough hard substrate. In this paper we have extended that study, and considered the spatula-plate adhesion.

Naturally occurring surfaces (e.g., a stone wall) have surface roughness on all length scales, from macroscopic to atomic. Adhesion between two bodies is only possible if the surfaces are able to deform (elastically or plastically) to make direct (atomic) contact at a non-negligible fraction of the nominal contact area. For "hard" solids this is nearly impossible and as a result adhesion is usually negligible between hard rough surfaces. ${ }^{11}$

The skin of the gecko toe pad is able to deform and follow the substrate roughness profile on length scales much longer than the thickness $d \approx 100 \mu \mathrm{m}$ of the elastic keratin film, say beyond $\sim 1000 \mu \mathrm{m}$. At shorter length scales the keratin film, because of its high elastic modulus (of order 1 $\mathrm{GPa}$ ), can be considered as rigid and flat. Elastic deformation of the pad surface on length scales shorter than $\sim 1000 \mu \mathrm{m}$ involves the compliant setae fiber array system, with fibers of thickness $\sim 4 \mu \mathrm{m}$. In Ref. 5 we have shown that if the surface roughness root-mean-square amplitude, measured over a patch $D \times D$ with $D \approx 1000 \mu \mathrm{m}$, is smaller than a characteristic length (the adhesion length) (see Ref. 5), then the fiber array system is able to deform (without storing a lot of elastic energy) to follow the surface roughness in the wavelength range $10<\lambda<1000 \mu \mathrm{m}$. However, if the setae fiber tips were blunt and compact, they would not be able to penetrate into surface "cavities" with diameters less than a few $\mu \mathrm{m}$. Thus, negligible atomic contact would occur between the surfaces, and the adhesion would be negligible. For this reason, at the tip of each long (thick) fiber an array of $\sim 1000$ thinner fibers (diameter of order $\sim 0.1 \mu \mathrm{m}$ ) occurs. These fibers are able to penetrate into surface roughness cavities down to length scales of a few tenths of a micrometer. However, if the thin fibers had blunt and compact tips made from the same "hard" keratin as the rest of the fiber, then one would still obtain very small adhesion, since a lot of elastic energy would be necessary to deform the surfaces of the thin fibers to make atomic contact with the substrate. Therefore, the top of the thin fibers end with thin, leaflike plates, which can easily be bent (without storing a lot of elastic energy) to follow the surface roughness profile. In Ref. 5 one of us speculated that the spatula tips are covered with a very soft compliant layer, e.g., a liquidlike (high mobility) layer of polymer chains grafted to the tip of the thin fibers. This liquidlike layer, if thick enough, would be able to adjust to the substrate roughness profile over lateral distances below $\sim 10 \mathrm{~nm}$. However, the calculation presented above (Figs. 10 and 11) indicate that such a layer may not always be necessary in order for strong adhesion to occur. However, the calculations presented in Fig. 6 show that, for rough surfaces with the fractal dimension $D_{\mathrm{f}}=3-H>2.2$, very small adhesion may occur in most cases.

Finally, we note that lizards are the heaviest living objects on this planet that are able to adhere to, e.g., a rough vertical stone wall. Since the surface area of a body increases more slowly than the volume (or mass) with an increase of
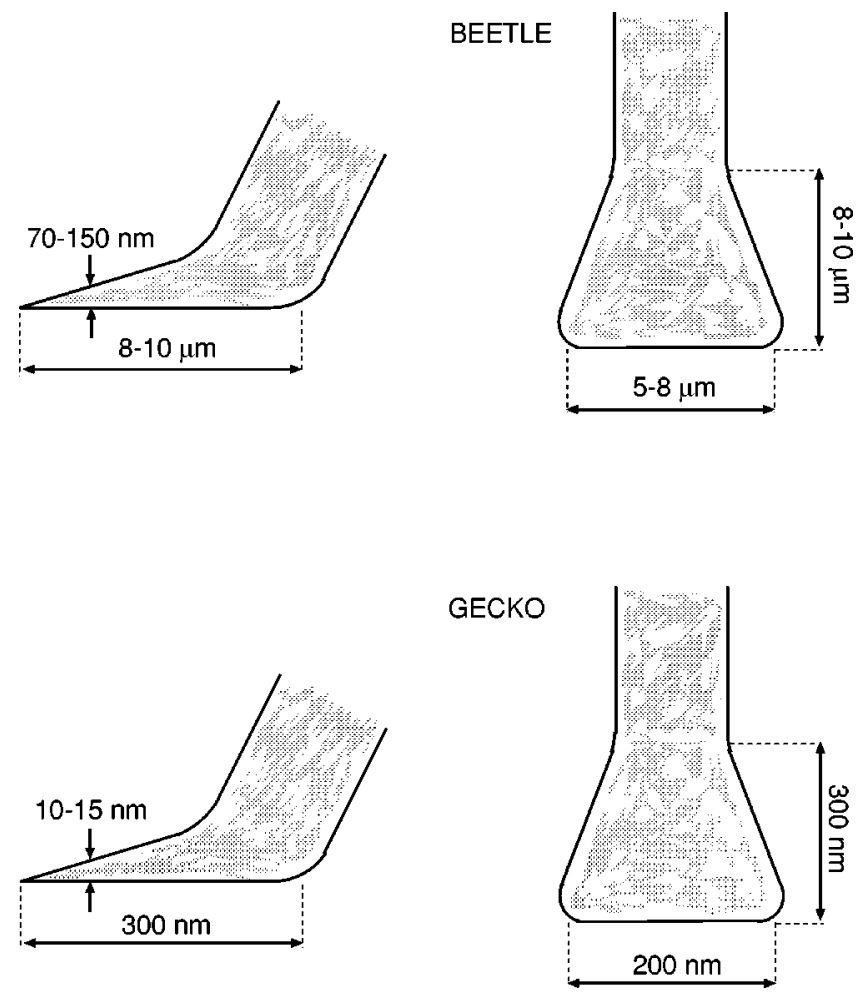

FIG. 14. The adhesive system of beetle and lizard. 
the linear size of the body, the adhesive system in large living bodies such as lizards must be more effective (per unit attachment area) than in smaller living objects such as flies or beetles. ${ }^{1}$ This implies that lizards have the most effective adhesive systems in the biological evolution for the purpose of locomotion. This is confirmed by electron microscopy studies. Let us compare the spatula of the adhesive systems of beetles with lizards (Fig. 14). Note that the spatula is thinner in lizards than in beetles. Also, the diameter of terminal branches is smaller. This implies that less elastic energy per unit surface area will be stored in the lizard adhesive system, and that the effective interfacial energy $\gamma_{\text {eff }}$ will be larger for lizards than for beetles.

\section{SUMMARY AND CONCLUSION}

We have studied the adhesion of elastic plates to rough substrates, which is relevant to biological systems, e.g., flies, crickets, and lizards, where the adhesive microstructures consist of a hierarchical array of thin fibers and plates. The effective elastic modulus of the fiber-plate arrays is very small on all relevant length scales (from $\mathrm{mm}$ to $\mathrm{nm}$ ), which is of fundamental importance for adhesion on rough substrates. We have shown how the adhesion depends on the nature of the substrate roughness, and applied the theoretical results to the adhesion pads of lizards. Experiments to test the theoretical results are underway. Finally, we note that the construction of manmade adhesives based on fiber and plate arrays may be an attractive alternative to the usual adhesives based on thin polymer films. Some pioneering experiments have indeed shown enhanced adhesion for fiber array sys- tems, but no manmade system of the hierarchical nature used in biological systems has so far been produced. ${ }^{12,13}$

\section{ACKNOWLEDGMENTS}

One of the authors (B.P.) thanks the EC for a "Smart QuasiCrystals" grant under the EC Program "Promoting Competitive and Sustainable GROWTH." S.G. wishes to thank A. Peressadko for AFM data. This work was supported by the Federal Ministry of Education, Science and Technology, Germany (Project BioFuture 0311851).

${ }^{1} \mathrm{M}$. Scherge and S. Gorb, Biological Micro- and Nano-Tribology (Springer, Berlin, 2001).

${ }^{2}$ B.N.J. Persson, Eur. Phys. J. E 8, 385 (2002); Phys. Rev. Lett. 89, 245502 (2002).

${ }^{3}$ K. Autumn, M. Sitti, Y.A. Liang, A.M. Peattie, W.R. Hansen, S. Sponberg, T.W. Kenny, R. Fearing, J.N. Israelachvili, and R.J. Full, Evolution, PNAS Early Edition; K. Autumn, Y.A. Liag S.T. Hsieh, W. Zesch, W.P. Chan, T.W. Kenny, R. Fearing, and R.J. Full, Nature (London) 405, 681 (2000); K. Autumn and A. Peattie, Int. Comp. Bio. 42, (2002); S. Sponberg, W. Hansen, A. Peattie and K. Autumn, ibid. 39, 395 (2002).

${ }^{4}$ K.N.G. Fuller and D. Tabor, Proc. R. Soc. London, Ser. A 345, 327 (1975).

${ }^{5}$ B.N.J. Persson, J. Chem. Phys. 118, 7614 (2003).

${ }^{6}$ L.D. Landau and E.M. Lifshitz, Theory of Elasticity (Pergamon, London, 1959).

${ }^{7}$ B.N.J. Persson and E. Tosatti, J. Chem. Phys. 115, 5597 (2001).

${ }^{8}$ J. Feder, Fractals (Plenum, New York, 1988).

${ }^{9}$ J.N. Israelachvili, Intermolecular and Surface Forces (Academic, London, 1995).

${ }^{10}$ B.N.J. Persson (unpublished).

${ }^{11}$ B.N.J. Persson, Sliding Friction: Physical Principles and Applications, 2nd ed. (Springer, Heidelberg, 2000).

${ }^{12}$ A. Peressadko and S.N. Gorb, J. Adhes. (to be published).

${ }^{13}$ A.K. Geim, S.V. Dubonos, I.V. Grigorieva, K.S. Novoselov, A.A. Zhukov, and S.Yu. Shapoval, Nature (London) 2, 461 (2003). 